\title{
Determining factors associated with inhaled therapy adherence on asthma and COPD: A systematic review and meta-analysis of the global literature
}

\author{
Constança Monteiro $^{a}$, Tiago Maricoto ${ }^{\mathrm{b}, \mathrm{c}, *}$, Filipe Prazeres ${ }^{\mathrm{a}, \mathrm{d}, \mathrm{e}}$, Pedro Augusto Simões ${ }^{\mathrm{a}, \mathrm{f}}$, \\ José Augusto Simões ${ }^{\text {a,e,g }}$ \\ ${ }^{\text {a }}$ Faculty of Health Sciences, University of Beira Interior, 6200-506, Covilhã, Portugal \\ ${ }^{\mathrm{b}}$ Aveiro Healthcare Centre, Aradas Health Unit, Aveiro, Portugal \\ ${ }^{\mathrm{c}}$ Faculty of Health Sciences, GRUBI - Systematic Reviews Group, University of Beira Interior, 6200-506, Covilhã, Portugal \\ d Family Health Unit Beira Ria, 3830-596, Gafanha da Nazaré, Portugal \\ e Centre for Health Technology and Services Research (CINTESIS), University of Porto, 4200-450, Porto, Portugal \\ ${ }^{\mathrm{f}}$ Pulsar Family Health Unit, Coimbra, Portugal \\ ${ }^{g}$ USF Caminhos do Certoma, do ACeS Baixo Mondego, Portugal
}

\section{A R T I C L E I N F O}

\section{Keywords:}

Adherence

Inhaled therapy

COPD

Asthma

\begin{abstract}
A B S T R A C T
Background: Adherence to therapy has been reported worldwide as a major problem, and that is particularly relevant on inhaled therapy for Asthma and Chronic Obstructive Pulmonary Disease (COPD), considering its barriers and features. We reviewed the global literature reporting the main determinants for adherence on these patients.

Methods: Searches were made using the Cochrane Library, MEDLINE, EMBASE and ISI Web of Science databases. Analytical, observational and epidemiological studies (cohort, case-control and cross-sectional studies) were included, reporting association between any type of determinant and the adherence for inhaler therapy on Asthma or COPD. Random-effects meta-analysis were used to summarise the numerical effect estimates.

Results: 47 studies were included, including a total of 54.765 participants. In meta-analyses, the significant determinants of adherence to inhaled therapy were: older age $\left[R R=1.07(1.03-1.10) ; \mathrm{I}^{2}=94 ; \mathrm{p}<0.0001\right]$ good disease knowledge/literacy $\left[R R=1.37(1.28-1.47) ; \mathrm{I}^{2}=14 ; \mathrm{p}=0.33\right]$; obesity $\left[\mathrm{RR}=1.30(1.12-1.50) ; \mathrm{I}^{2}=0 ; \mathrm{p}\right.$ $=0.37]$; good cognitive performance $\left[\mathrm{RR}=1.28(1.17-1.40) ; \mathrm{I}^{2}=0 ; \mathrm{p}=0.62\right]$; higher income $[\mathrm{RR}=1.63$ (1.05-2.56); $\left.\mathrm{I}^{2}=0 ; \mathrm{p}=0.52\right]$; being employed $\left[\mathrm{RR}=0.87(0.83-0.90) ; \mathrm{I}^{2}=0 ; \mathrm{p}=0.76\right]$ and using multiple drugs/inhalers $\left[\mathrm{RR}=0.81(0.79-0.84) ; \mathrm{I}^{2}=0 ; \mathrm{p}=0.80\right]$. Overall, the strength of the underlying evidence was only low to moderate.

Conclusions: Many determinants may be associated to patient's adherence, and personalised interventions should be taken in clinical practice to address it by gaining an understanding of their individual features.
\end{abstract}

\section{Introduction}

Asthma and Chronic Obstructive Pulmonary Disease (COPD) are chronic inflammatory disorders of the airways characterised by airway obstruction and affects approximately $10 \%$ of the global population. The most recent recommended therapy according to major guidelines is through inhaled therapy [1,2].

According to previous reports [3] the concept of adherence emphasizes the need for agreement and can be defined as the extent to which the patient's behavior matches the agreed recommendations from the prescriber and the prescription. To achieve optimal adherence, a patient must undertake a sequence of key steps (initiation, implementation and persistence/discontinuation), and each one of them has specific related features and may be influenced by different factors $[4,5]$. Adherence to pharmacological treatment is, therefore, a key factor to control chronic respiratory diseases. However, many studies reveal poor adherence to the therapy in all pathways, either oral or inhaled, which leads to poor disease control. This is particularly relevant on asthma and COPD

\footnotetext{
Abbreviations: COPD, Chronic Obstructive Pulmonary Disease; GINA, Global Initiative for Asthma; GOLD, Global Initiative for Chronic Obstructive Lung Disease.

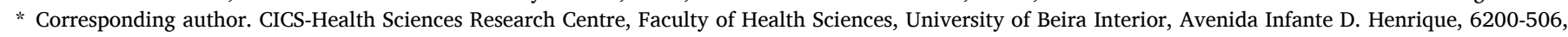
Covilhã, Portugal.

E-mail address: tiago.maricoto@gmail.com (T. Maricoto).
} 
patients, considering that, to achieve the best therapeutic efficacy, patients must properly adhere to the prescribed regiment, but also, use their inhalers correctly and with appropriate inhalation technique $[6,7]$. In fact, inhaler adherence and inhalation technique are close, but quite independent concepts, and different factors may influence each one of them. Nevertheless, some studies highlight the role of inhaler poor adherence as it may contribute to inhalers misuse and poor inhalation technique [8]. These patients are exposed to higher risks of adverse outcomes and to significant morbidity and mortality.

Non-adherence to inhaled therapy may be associated with several factors, either intentional (linked to patients willing to take it or even to patients' beliefs) or unintentional (linked to patients sociodemographic and other objective features, and that may affect their ability to use inhalers properly and without inhaler technique errors). Some studies have pointed out the main factors of therapeutic adherence, such as its associated costs, patient's educational level, age, adverse side effects, social and economic issues, satisfaction with treatment, symptomatic control and improvement in lung function, complexity of inhaler functioning, among others [9]. In fact, non-adherence may be related to factors at several levels, such as the patient level (including intentional and non-intentional drivers that affect patient's motivation and ability to adhere), the patient-provider level (related to communication and to the prescribing process) and the healthcare system level (related to the access to medication) [10,11].

Inhaled therapy presents significant levels of non-adherence [12] leading to increased exacerbations and consequently to increased risk for hospital admissions. There is still an ongoing discussion and controversy, upon which predictors are most relevant to inhaler adherence [13]. Previous works conducted with asthma patients identified intentional [14] and unintentional [15] determinants, but found inconsistent results, mainly due to the heterogeneity of instruments and operational measures of adherence, as well as patient's characteristics. Also, a previous systematic review addressing interventions to improve adherence pointed out the same limitations [16]. Furthermore, previous systematic reviews focused mostly on asthma patients, but non-adherence to inhaled therapy equally affects COPD patients, and those also need to be addressed in future studies $[8,9,13,14,17]$.

A systematic review was performed aiming to assess the most relevant determining factors associated with poor inhaler adherence in patients with asthma or COPD.

\section{Methods and analysis}

A protocol for this study was developed, registered with the International prospective register of systematic reviews (PROSPERO) (registration number CRD42020167249) [18]. This review is reported in accordance with the PRISMA guidelines for systematic reviews and MOOSE guidelines for meta-analysis of observational epidemiological studies $[19,20]$. No modifications were made, compared with the initial protocol.

\subsection{Eligibility criteria for study selection}

\subsubsection{Participants and study types}

Were included all studies that had participants of any age, with diagnosed of Asthma and/or COPD, designed as observational and analytical epidemiological studies, including cohort, case-control and cross-sectional studies. Were excluded discussion papers, non-research letters and editorials, randomised control trials, clinical case studies and case-series, and animal studies.

\subsubsection{Exposure}

Studies that include any demographic, individual, psychosocial and environmental predictors of inhaled therapy adherence were eligible for inclusion.

\subsubsection{Study outcomes}

The outcome was the effective adherence to the inhaled therapy, measured either on a validated scale or by other mean that the authors may have found suitable.

\subsection{Search strategy}

A comprehensive search strategy was developed for retrieving published and unpublished studies on the topic (online supplementary Appendix S1 - "Search strategy"). Searches were made in the Cochrane Library, MEDLINE, EMBASE and ISI Web of Science. Search dates were from inception up to October 2020. The references in all eligible studies were reviewed to identify additional studies. No language restrictions were imposed in the searches and translations were made where necessary.

\subsection{Selection process}

Papers retrieved from the databases were exported to the online reference management software Rayyan ${ }^{\circledR}$ (available at rayyan.qcri.org). Two reviewers (CM and TM) independently selected the articles according to the defined criteria and applied the following screening stages: cleaning of duplicated articles, selection of articles according to eligibility criteria and by reading the title and abstract, and selection of articles according to full text reading. All disagreements were resolved through discussion or arbitrated by a third review author (JAS).

Reasons for excluding articles during the full text screening were noted and indicated in PRISMA diagram (Fig. 1) [21].

\subsection{Data collection process}

Two authors (CM and TM) collected data from included articles in their original presentation and recorded them in a spreadsheet made in Microsoft Excel software and tailored to the current systematic review. Indirect data from figures and charts was collected, adapting their interpretation by consensus, and contacted authors of original articles for further information and data when necessary. Any disagreement in data collection was resolved through discussion or arbitrated by a third review author (JAS).

\subsection{Type of data collected}

The following information from all included studies was collected:

- Study design, number of participants and their characteristics (age, gender, type of diagnosis, years since diagnosis and asthma or COPD stage/grade), variables of exposure to determinants/predictors (such as smoking, lifestyle habits, environmental exposures, cognitive performance, comorbidities such as obesity, diabetes or cancer, clinical control, exacerbation history, employment, literacy, educational level, income, $\mathrm{n}^{\circ}$ of outpatient/regular visits and other psychosocial or objective determinants ...), inhalers used (type of device, inhaler performance, previous inhaler performance education received, type of drugs used), follow-up (in case of cohort studies), country of study, year of publication

- Estimates of the association between parameters of the main determinants/predictors identified and the study outcome (measured as hazard ratio, risk ratio, odds ratio, 95\% confidence intervals, mean and SD). One author (TM) inserted data into Review Manager Software (RevMan) (available at http://community.cochrane.org), and data were double-checked for correct entry by a second author (CM).

\subsection{Quality assessment}

Two authors (CM, TM) appraised the quality of included studies using an adaptation of the GRADE quality assessment tool [22,23]. 


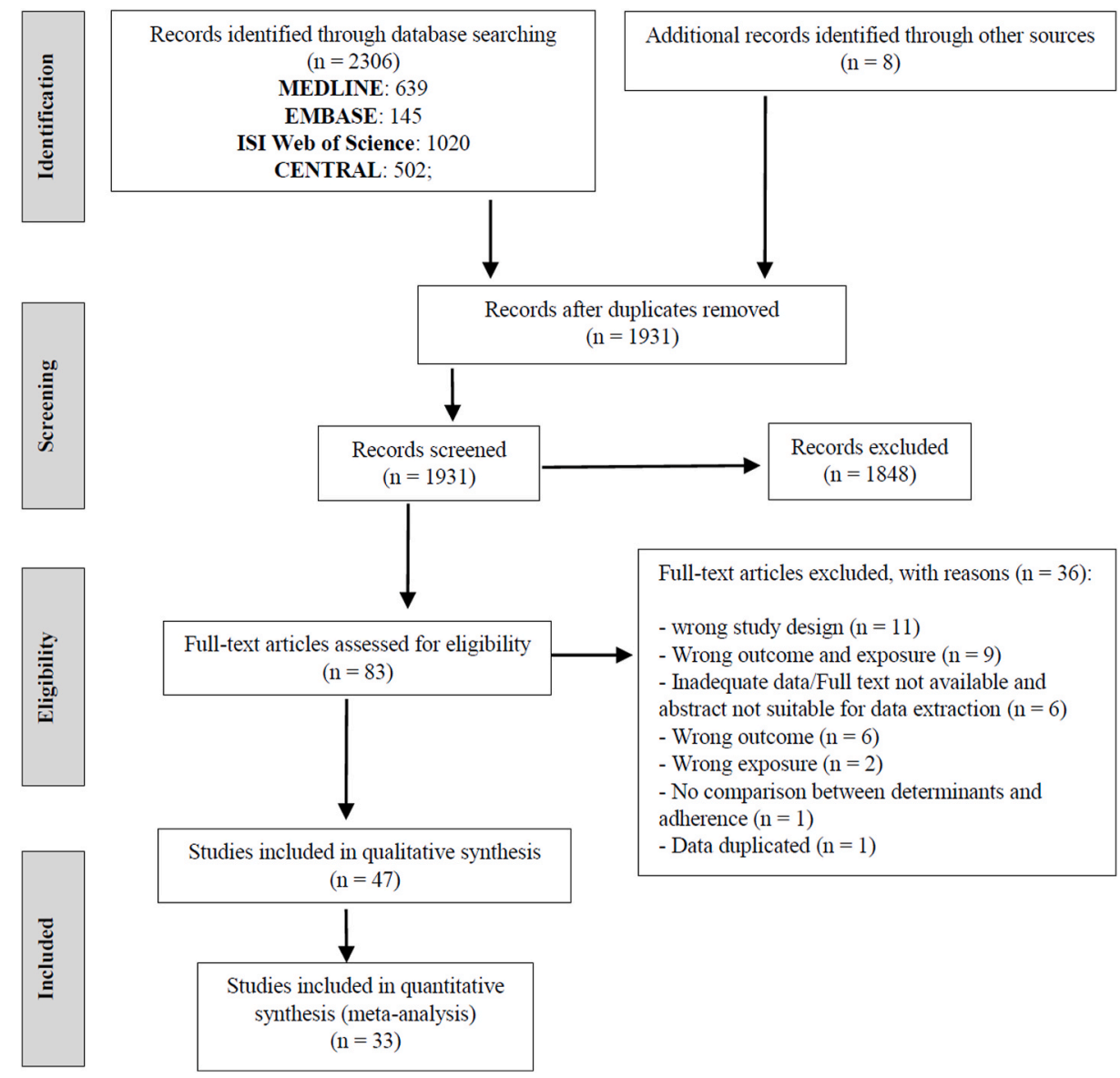

Fig. 1. Flow diagram on search and article inclusion, according to PRISMA statement.

Different components of each study were appraised, including confounding, selection, measurement of exposure, departures from exposure, missing data, measurement of outcomes and reported results. For each study, the grading of each individual components and the global study rating were assigned categories of risk of bias: low, moderate, severe, and critical. The global grading involved taking an average and relative weight of all individual components. Any disagreements not resolved by discussion were arbitrated by a third reviewer (JAS).

\subsection{Data synthesis}

All variables regarding the determinants and the outcome measure were collected in their original status, and the ones considered similar enough to be combined in quantitative analysis were used for metanalysis. Regarding adherence, all collected studies reported it on a continuous or dichotomous format, but only those reporting it as a dichotomous variable were able to be included in the metanalysis. It was possible to categorize the different methods to measure adherence in four main types but not to standardize the different cut-offs that were used among the original studies to dichotomize it. Therefore, regarding the prevalence of "good adherence" and its association to the potential determinants. Raw data was used as it is reported by original studies.

A descriptive summary of all included studies is presented in order to summarise literature. All studies were judged to be reasonably, clinically and methodologically heterogeneous. Therefore, meta-analyses were performed using random-effects models. The heterogeneity between studies was quantified using the I2 statistic. Sensitivity analyses were made on the basis of risk of bias in the studies in order to assess the robustness of these findings to different assumptions. In the metaanalysis, estimates from studies not presented as RRs, were converted to RRs using the recently proposed formulae provided by VanderWeele et al. [24] Sub-group analyses were performed, for all determinants, according to: publication year (by decades), continental region, study design (cross-sectional or cohort), risk of bias assessment, study dimension/size, diagnosis type (asthma, COPD or both), types of instruments to measure adherence (dose counting or eletronically monitored, or via an unspecific self-reported measure, Morisky Medication Adherence Scale (MMAS) or Test of Adherence to Inhalers (TAI) and study participants mean ages). Publication bias was accessed using funnel plots. The meta-analyses were performed using Cochrane Review Manager Software@ (available at http://community.cochrane.org). The PRISMA checklist was followed for reporting of the systematic review.

\section{Results}

\subsection{Description of studies}

The research yielded 2306 articles, and, after elimination of duplicates, 1931 remained. From these articles, 1848 were excluded after reading title and/or abstract. Thus, 83 studies were obtained, from which, after reading the full text, 36 were excluded for several reasons (Fig. 1).

Of the 47 eligible studies [3,25-70], 21 (44.7\%) were cross-sectional and $26(55.3 \%)$ were cohort studies. A total of 54.765 individuals were studied and most $(\mathrm{n}=41 ; 87.2 \%)$ were studies in adults.

Twenty-eight studies (59.6\%) included only Asthma patients, and 11 (23.4\%) only COPD patients. Although the majority of studies did not report data about disease severity, those that did, included participants with moderate and poorly controlled Asthma or COPD.

Detailed information for all selected studies is available at 
supplementary Appendix S3 - "Complete data of selected studies".

\subsection{Measures of adherence}

Several tools were used to measure adherence to inhaled therapy. Most studies ( $\mathrm{n}=23 ; 48.9 \%)$ used a dose counting or electronically monitored systems, while 9 studies (19.2\%) used an unspecific selfreported measure; 5 (10.6\%) used the Morisky Medication Adherence Scale (MMAS) and other 5 (10.6\%) used the Test of Adherence to Inhalers (TAI).

Among studies using "dose counting or electronically monitored systems", we found one study measuring adherence through a direct dose counting on inhaler (61), the majority using a community or hospital pharmacy electronic record system $(47,48,56,44,65,28,39,37$, $21,53,29)$ and 5 using a smart-inhaler or an electronic monitor attached to the inhaler $(26,63,27,59,55)$. Most of them defined good adherence as a dichotomous variable, considering a compliance of at least $70-80 \%$ among the prescribed days or doses. The exception were 2 studies (37, 21 ) that defined the cut-off at $50 \%$, and 2 others $(53,29)$ that defined it as "taking medication in a regular/daily basis". 3 studies $(26,63,27)$ did not specified the cut-off criteria used for good adherence.

All the studies using the Morisky Medication Adherence Scale (MMAS) defined adherence using the predefined and validated cut-off for a dichotomous variable setting of "good adherence", although one study used de 4-item version (38) and other de 8-item version (43). The same coherence was found in studies using Test of Adherence to Inhalers (TAI), and studies using Medication Adherence Reporting Scale (MARS), except for 2 studies that used the 10-item version of MARS $(49,57)$.

Several studies did not report any specific and objective measure of adherence, resorting to patients self-reporting. Nevertheless, some of them $(31,32,50)$ defined good adherence if participants reported a compliance of at least $80 \%$ among the prescribed days or doses, or $80 \%$ positive answers to some type of unstandardized questionnaire.

\subsection{Risk of bias in included studies}

Two reviewers (CM and TM) independently evaluated the risk of bias of the included studies, reaching consensus in all evaluations (Fig. 2). Most studies showed a predominance of moderate to serious risk of bias in their risk assessment. Among 47 studies included in quality assessment, only $5(10.6 \%)$ had a global low risk of bias and 7 (14.9\%) were considered critical. The dimension found to have the highest risk of bias concerned measurement of outcomes and missing data (detailed evaluation in supplementary Appendix S2 - "Quality Assessment and Risk of Bias of included studies").

\subsection{Determinants of adherence}

The determinants of adherence mostly addressed were: age, gender, smoking habits, professional situation, disease knowledge/literacy, education, time since diagnosis, outpatient clinic visits, comorbidities (such as obesity, diabetes or cancer), history of past exacerbations, drugs and inhalers used, cognitive performance, disease severity and family income.

Thirty-three studies were eligible for data extraction, reporting data from 44908 participants, and allowing the conduction of meta-analysis of the association between determinants and the risk good adherence to inhaled therapy. Detailed information on meta-analysis for each main determinant of interest is available at supplementary Appendix S4 "Complete data meta-analysis".

Overall, significant trends were observed towards an increased or decreased risk for adherence. Fig. 3 reports the main findings from the performed meta-analysis. Therefore, the following determinants revealed to be significantly associated to good adherence on the pooled estimations: older age $\left[\mathrm{RR}=1.07(1.03-1.10) ; \mathrm{I}^{2}=94 ; \mathrm{p}<0.0001\right]$; having good disease knowledge/literacy $\left[\mathrm{RR}=1.37\right.$ (1.28-1.47); $\mathrm{I}^{2}=$ 14; $\mathrm{p}=0.33$; obesity [RR = $\left.1.30(1.12-1.50) ; \mathrm{I}^{2}=0 ; \mathrm{p}=0.37\right]$; having a good cognitive performance $\left[R R=1.28(1.17-1.40) ; \mathrm{I}^{2}=0 ; \mathrm{p}=0.62\right]$ and having a higher income $\left[R R=1.63(1.05-2.56) ; I^{2}=0 ; p=0.52\right]$. On the other hand, the following determinants were associated to a poor predictive adherence: being employed $\left[R R=0.87(0.83-0.90) ; \mathrm{I}^{2}=0 ; \mathrm{p}\right.$ $=0.76]$ and using multiple drugs/inhalers $\left[\mathrm{RR}=0.81(0.79-0.84) ; \mathrm{I}^{2}=\right.$ $0 ; \mathrm{p}=0.80]$.

Subgroup analysis revealed that smoking exposure (active, past or passive) is associated with good adherence on European studies only $\left[R R=1.32(1.10-1.59) ; I^{2}=0 ; p=0.50\right]$. Previous exacerbations revealed to be associated with better adherence on one single studies with Asthma patients, developed on North America $[R R=1.52$

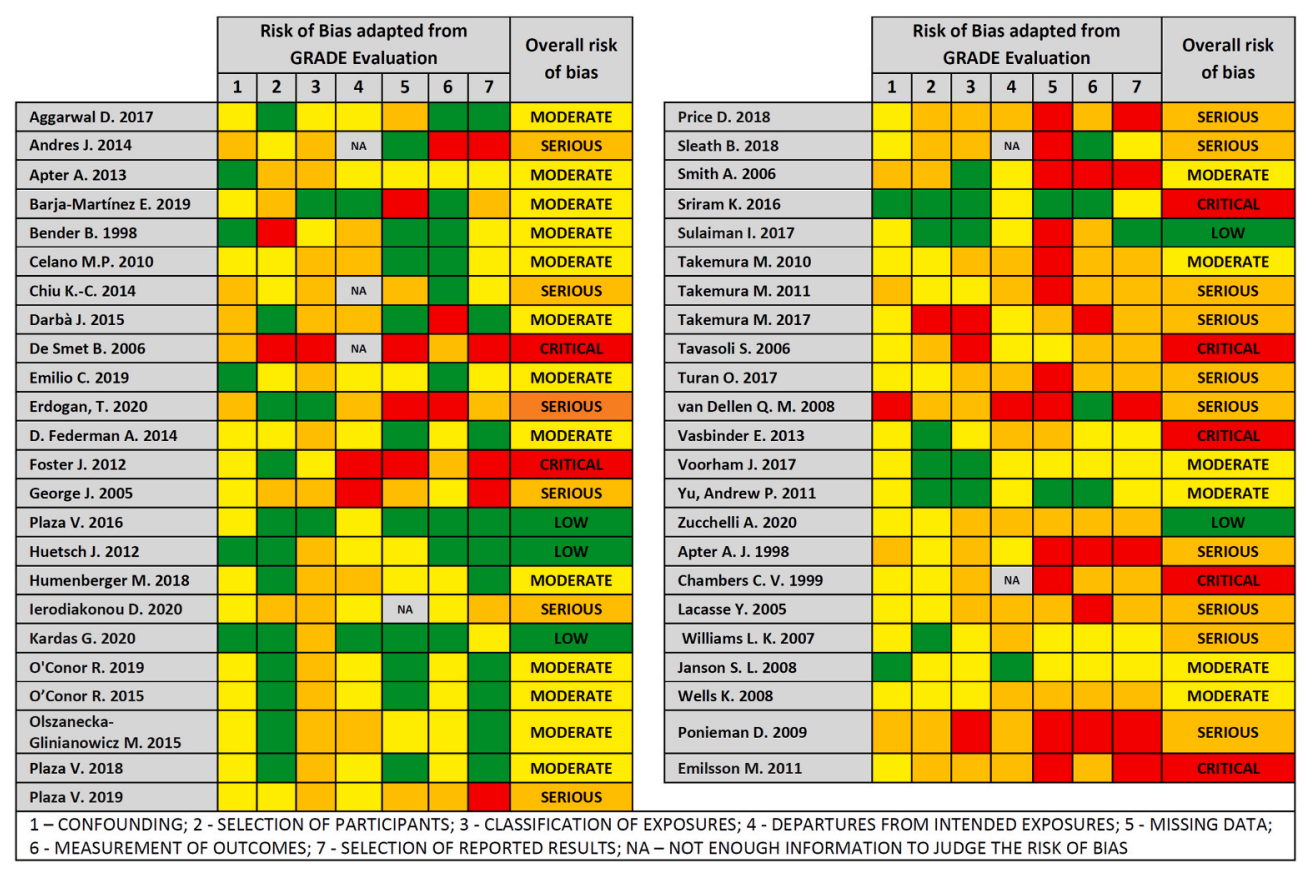

Fig. 2. Risk of Bias assessment in included studies according to an adaptation of the GRADE quality assessment tool. 


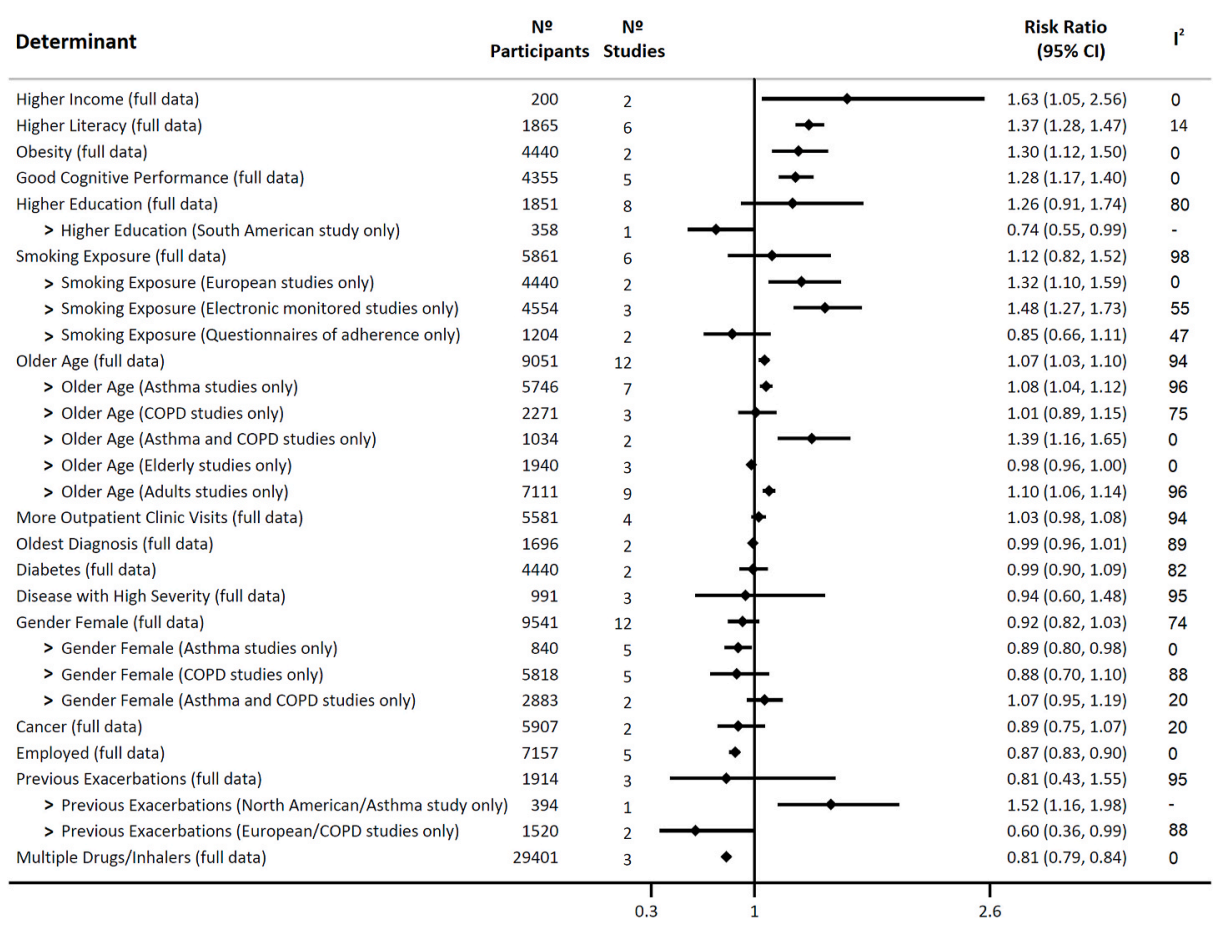

Fig. 3. Forest plot of results on the risk of good adherence to inhaled therapy according to the reported determinants and the most relevant sub-group analysis. All determinants are ordered by their RR mean estimate, except for specific sub-group analysis that revealed statistically relevant estimates (signalled with the arrowhead mark). $\mathrm{I}^{2}$ - Heterogeneity.

(1.16-1.98)], but with poor adherence in two studies performed in Europe with COPD patients [RR $\left.=0.60(0.36-0.99) ; \mathrm{I}^{2}=88 ; \mathrm{p}=0.003\right]$. Also, regarding "older age" as a determinant for good adherence, that was found mostly in studies performed in adult populations, while in elderly populations the trend was the opposite but not significant.

Additional sensitivity analysis revealed no significant changes of the risk estimates regarding other variables of interest, such as for publication year, study design, risk of bias assessment, study dimension/ sample size, diagnosis included, or types of instruments used to measure adherence. Detailed date is available at supplementary Appendix S3 "Complete data of selected studies".

No data were available to perform subgroup analysis according to different types of inhalers or therapies used, adherence behaviour assessed (initiation, implementation or persistence) and adherence interface level (patient, provider or health service).

\section{Discussion}

\subsection{Summary of key findings}

Adherence to therapy has been a challenge for many years, and several factors have been reported to affect adherence on chronic conditions [71,72]. This systematic review is the first to address, at a global, comprehensive, and integrated level, the main determinants of adherence to inhalation therapy in patients with COPD and/or Asthma, and to quantify the associated risks.

These results show that the major determinants associated with better adherence were higher income $(63 \%$ in mean relative risk increase), high degree of literacy (37\% increase), obesity (30\% increase), good cognitive performance ( $28 \%$ increase), and older age $(7 \%$ increase). On the other hand, being employed was associated with a decreased risk for adherence ( $23 \%$ in mean relative risk decrease) as well as using multiple drugs/inhalers (29\% decrease). In addition, subgroup analysis revealed that smoking habits are also associated with an increased risk for adherence in European countries (32\% increase), and the gender woman with lower adherence in Asthma (21\% decrease).
Regarding the presence of previous exacerbations, in Asthma it might be associated with better adherence, while in COPD it might be associated with lower adherence.

One study reported a greater adherence in dry powder inhaler users compared to pressurized metered dose inhaler users, however, it was not sufficient to be included in the meta-analysis [57].

Nevertheless, no relevant influence on the risk for better adherence was found considering professional situation, education, time since diagnosis, number of outpatient clinic visits, having other comorbidities (such diabetes or cancer) or disease severity stage.

\subsection{Strengths and limitations of the review}

Throughout this review no significant inequities between most of the studies were found, despite the high methodological heterogeneity and the diversity of applied scales among them. Most included studies have poor methodological quality and a high risk of bias, which places a limitation on the confidence in the reported results. Another major limitation was the heterogeneity present in the scales and instruments used to measure adherence, as well as to report and measure the determinants.

Considering adherence itself, different methods were used to measure and define it. Most used objective measures and defined "good adherence" in a dichotomous way, to establish its association to the potential determinants. We highlight the fact that the majority of studies defined good adherence as "never or rarely forgetting to take a medication", with similar cutoffs applied (about 70-80\%), either to validated questionnaires, electronic records or self-reporting unspecific scales. Therefore, we find it less likely to consider a significant bias upon this definition.

Moreover, the methods used to define the determinants were also variable. This variety, as well as the high standards defined for "good adherence", means that the reported risk estimations may, at some extent, not faithfully represent the environment in the "real world". This may also explain the significantly high heterogeneity found in most pooled estimations as saw in the $\mathrm{I}^{2}$ values. As an example, age was 
reported by several studies as a dichotomous variable using different cutoffs, such as 50, 60 or 65 years; therefore, a single cutoff for the pooled risk estimation can not be established. That may be the reason why "older age" was detected as a significant predictor for better adherence in studies with adult populations, while in studies with elderly participants the trend was not significantly different. For such populations, other factors may also be involved in adherence beside objective and sociodemographic characteristics [11]. Moreover, obesity was reported only by two studies as being associated to adherence, but only one study [43] defined it objectively as body mass index $\geq 30$ $\mathrm{kg} / \mathrm{m} 2$ or by the presence of a clinical diagnosis on the participant's record. There is though the doubt whether a slighter overweight may also influence adherence patterns, or even if obesity is playing a role as a surrogate or confounding for adherence, considering that such patients may at some extent, need for more (and be more exposed) to accurate and persuasive clinical and educational interventions. The same limitation may be applied to family income, where most studies used the national mean family income as the main cutoff, but this may differ significantly across different countries. Cognitive performance for instance, was defined in most studies in a homogeneous way, as the presence/absence of cognitive impairment, but different scales were used for cognitive assessment, such as Mini Mental State Examination, Montreal Cognitive Assessment Test and other type of skills and memory tests. Other determinants were reported with a better homogeneity, such as higher educational attainment, which was defined in almost every study with the cutoff in the secondary school level.

Additionally, many studies did not assess the association between determinants and adherence with multivariate analysis techniques, nor were adjustments made to confounding factors, leading to a large bias in the obtained results. For this reason, the estimations resulted in a combination of adjusted and unadjusted risk ratios.

However, the methodological quality applied throughout the review must be highlighted, in accordance with the PRISMA recommendations (Preferred Reporting Items for Systematic Reviews and Meta-Analysis) [21] and compelling on a well-structured method. A broad, but concrete, study inclusion criteria (PICO) was designed, reaching consensus among all reviewers on a rigorous way, and achieving a careful final approach to the results. The inclusion of broad criteria allowed to develop a highly comprehensive review with a significant diversity of determinants included, with more generalised results. In addition, regardless of the limitations pointed out, some of the pooled results revealed significant risk associations, either with precise confidence intervals, but also of great magnitude of effect, which reinforces the confidence of such estimates. Studies in different languages were also included, to prevent the imposition of a linguistic barrier and bias.

Overall, there were significant heterogeneity on the studies methodologies and some estimates was based on few studies, for which the risk of bias is moderate to severe. Nevertheless, some determinants revealed narrow estimates and strong magnitude on their pooled risk ratio, such as "literacy", "being employed" and "cognitive performance". Considering that, the overall confidence of the results is low, but for these specific determinants it may be moderate.

\subsection{Comparison with previous studies}

Inhaled therapy adherence is a topic with limited available studies so far [73]. For that reason, we find this review relevant and timely, addressing this important gap.

In the scope of inhalation therapy, previous work focused on aspects related to users' "beliefs" (psychological and subjective determinants). However, it does not address all other sociodemographic determinants that are considered to be key issues that affects patient adherence [14]. In the present review, there were also several studies addressing aspects of a more subjective character, such as beliefs about medications and patients' perceptions about diseases. Yet, their characteristics and the data reported hampered the ability to include them in the meta-analysis.
One study demonstrated that perceptions of behavior are better predictors than sociodemographic ones, reinforcing the paramount diversity of factors that should be considered in this type of reviews [74].

Another review, on Asthma patients, ended up detecting only age as a relevant determinant of adherence. Authors point out the significant heterogeneity of the included studies as the main limitation [15]. This limitation is also highlighted in the present review. Nevertheless, its scope is broader because it included studies on Asthma and COPD and reported approximate risk estimations. In fact, in COPD, the patients' characteristics and the used medication are also considered to be key factors of adherence to therapy, as revealed by previous works [75].

Some interventional studies (with ICS only) tried to improve adherence, but without effective results. This might be due to external barriers and other key determinants [76] as highlighted by this work.

GINA and GOLD guidelines have been, since many years, pointing out the importance of patients' determinants on the risk for inhaled therapy adherence [1,2]. However, they are unspecific and don't consider such determinants in a global context, regardless the inhaler technique used. Some of those highlighted determinants are the type and regimen of medication, the difficulty in handling the device due to pathologies such as arthritis, the frequency of intake on the same day, and the use of different/multiple devices. They also mention the importance of unintended factors (e.g. the poor understanding of the instructions given to patient, forgetfulness, the absence of a daily routine and the cost), but also intentional factors (e.g. the perception that treatment is unnecessary, the denial of asthma or its treatment, inappropriate expectations, concerns about adverse effects, dissatisfaction of caregivers, stigma, cultural and religious issues and costs). GOLD also points out the use of multiple devices, older age and the lack of education on inhaler performance as key factors for COPD inhaled poor adherence.

\subsection{Interpretation and implications of the findings}

Further research must be carried out to clarify the influence of sociodemographic and psychological determinants on adherence to inhaled therapy, mainly to access their synergic or collinear effects on a long-term scope. Some of those factors may at some extent, affect the patient's behavior regarding the disease and its outcomes. That may explain the influence found in our review between "having previous exacerbations" and a better adherence in Asthma, but worse adherence in COPD. Previous works have also found a similar trend in Asthma [77], and, therefore, future studies should address this potential causal relationship. Moreover, ageing itself may have a different effect on adherence during adulthood (improving it) then compared to geriatric age (decreasing it), and therefore needs further research.

These results highlight the need to improve clinical guidelines and practice, in order to address such determinants in patients with Asthma and COPD, thus, increasing the rates of adherence to inhalation therapy. This review brings new evidence to be considered and, possibly, to be included in an upcoming review of GINA and GOLD guidelines, where new sociodemographic determinants should be considered.

Another relevant aspect that may be addressed in the future is to distinguish between factors associated with non-adherence at the patient, provider, and system level, as well as at different temporal stages (initiating, implementing, and persisting). The studies performed so far have not provided enough information to distinguish such interface levels from each other, as all the reported estimations were at a patient level and patient centered. Nevertheless, some determinants for adherence hereby reported are more related to patients, and others to providers or to the system/society itself, but no confident conclusions can be established about their distinction.

Further studies should be carried out in developing countries. The scarcity of research in these countries is delaying the implementation of effective interventions and adherence measures, where poor adherence prevails due to the fact that the determinants in these populations are 
not certainly known [78].

Subsequently, it would be important to assess other specific adherence determinants, such as the type of inhaler used (dry powder inhaler versus pressurized devices) [57], or even different patterns between Asthma and COPD patients. The same applies to employment (hereby associated with a decreased risk for adherence), where private insurance-based healthcare systems may play a different role than compared to those based on national social health services [79]. Also, smoking habits have been identified to be related to adherence, but previous studies found opposite results, and this has been pointed out as a potential confounding effect, as smoking may be a proxy for other related behavioral and lifestyle aspects (i.e. anxiety) [80,81].

At the methodological level, previous studies have also pointed out the need for an harmonisation and padronisation of the exposure and outcome assessment, namely the tools used to measure adherence, but also the way that all determinants are measured and reported [15]. This will allow future systematic reviews to carry out more accurate pooled risk estimations. Several methods may be used to measure adherence, such as through pill counting, self-report, pharmacy refill accounting, electronic monitoring, measurement of blood levels of drugs and even frequent observation by healthcare professionals [82]. Nevertheless, some of them are complex or have significant costs, and therefore, there is the need for a more global, uniform, and consensual method. In this systematic review no relevant differences were found on the risk associations of all determinants, when comparing different methods to measure adherence. At some extent this points out the need for future studies comparing different methods to access adherence in real world.

Future studies should be designed on a longitudinal cohort base, with long-term follow-up, in order to assess these causal relationships over time, to control for potential confounders, and to access the risk of synergic or collinear effects. Also, psychological and cultural factors must be addressed in future studies, considering their important dimensions in the holistic approach to patients adherence [83].

This systematic review presents a wide, inclusive, and comprehensive approach, including studies from all over the world and settings, and therefore we find the results timely and generalizable to real world. This highlights the importance for clinicians to focus their interventions on key factors of adherence to achieve optimal efficacy, regardless of their context.

Finally, by identifying factors related to adherence, this systematic review highlights the need for future interventions that may ultimately modify them, such as employment, literacy, obesity, cognitive performance, and this should be addressed not only by future interventional studies, but also on a national and global scale of health policies.

\section{Conclusion}

Adherence to inhaled therapy is the cornerstone of Asthma and COPD clinical control and some determinants may be significantly associated with better adherence, such as higher income, high degree of literacy, obesity, good cognitive performance and older age. On the other hand, being employed and using multiple drugs/inhalers was associated with decreased adherence. Smoking patterns and gender may also play an important role, but uncertainty remains.

Asthma and COPD guidelines should give a reinforced attention to these determinants, recommending their assessment in every appointment. Further longitudinal, well-designed cohorts with long-term follow-up should be developed in order to clarify such causal effects.

\section{Author contributions}

This study was designed by CM and TM. Data extraction was performed by CM and TM. Data review and analysis was performed by all authors, but statistical tests were performed in first approach by TM. The first draft was written by CM and TM. All authors commented on the first draft and agreed with the final version. JAS is the guarantor of the study.

\section{Ethical considerations}

This work was not submitted to ethical board approval, considering it in a systematic review, and therefore, there was no human participation, and no personal data was used.

\section{Data sharing}

All data used in this systematic review is detailed and available at Supplementary Appendices.

\section{Sources of support}

This work was developed without any funding support or financial source. The academic affiliation of this systematic review is the Faculty of Health Sciences at the University of Beira Interior in Portugal.

\section{Differences between protocol and review}

The protocol of this systematic review was registered in PROSPERO with the number CRD42020220805, available at:

https://www.crd.york.ac.uk/prospero/display_record.php? ID $=$ CRD42020220805.

The study protocol has no differences from the final work.

\section{Prospero protocol registration $\mathrm{N}^{\mathrm{O}}$}

CRD42020220805.

\section{Declaration of competing interest}

The authors declare no conflict of interests.

\section{Acknowledgments}

None to declare.

\section{Appendix A. Supplementary data}

Supplementary data to this article can be found online at https://doi. org/10.1016/j.rmed.2021.106724.

\section{References}

[1] Global Initiative for Asthma, Global initiative for asthma: global strategy for asthma management and prevention (updated 2020), Rev. Fr. d'Allergologie d'Immunologie Clin. 36 (6) (2020) 685-704, https://doi.org/10.1016/S0335-7457 (96)80056-6.

[2] Gold, Global Initative for Chronic Obstructive Lung Disease, 2021.

[3] M. Emilsson, et al., The influence of personality traits and beliefs about medicines on adherence to asthma treatment, Prim. Care Respir. J. 20 (2) (2011) 141-147, https://doi.org/10.4104/pcrj.2011.00005.

[4] B. Vrijens, et al., What we mean when we talk about adherence in respiratory medicine, J. Allergy Clin. Immunol. Pract. 4 (5) (Sep. 2016) 802-812, https://doi. org/10.1016/j.jaip.2016.05.019.

[5] B. Vrijens, et al., A new taxonomy for describing and defining adherence to medications, Br. J. Clin. Pharmacol. 73 (5) (May 2012) 691-705, https://doi.org/ 10.1111/j.1365-2125.2012.04167.x.

[6] W. Vincken, R. Dekhuijzen, P. Barnes, The ADMIT series - issues in Inhalation Therapy. 4) How to choose inhaler devices for the treatment of COPD, Prim. Care Respir. J. 19 (1) (Nov. 2009) 10-20, https://doi.org/10.4104/pcrj.2009.00062.

[7] M.E. Broeders, J. Sanchis, M.L. Levy, G.K. Crompton, P.R. Dekhuijzen, The ADMIT series - issues in Inhalation Therapy. 2) Improving technique and clinical effectiveness, Prim. Care Respir. J. 18 (2) (May 2009) 76-82, https://doi.org/ 10.4104/pcrj.2009.00025.

[8] T. Maricoto, D. Santos, C. Carvalho, I. Teles, J. Correia-de-Sousa, L. TabordaBarata, Assessment of poor inhaler technique in older patients with asthma or COPD: a predictive tool for clinical risk and inhaler performance, Drugs Aging 37 (8) (2020) 605-616, https://doi.org/10.1007/s40266-020-00779-6.

[9] M.J. Mäkelä, V. Backer, M. Hedegaard, K. Larsson, Adherence to inhaled therapies, health outcomes and costs in patients with asthma and COPD, Respir. Med. 107 (10) (2013) 1481-1490, https://doi.org/10.1016/j.rmed.2013.04.005. 
[10] C.A. Horne R, V. Cooper, V. Wileman, Supporting adherence to medicines for longterm conditions: a perceptions and practicalities approach based on an extended common sense model, Eur. Psychol. 24 (1) (2019) 82-96, https://doi.org/ 10.1027/1016-9040/a000353.

[11] Y. Yap, A.F. Thirumoorthy, T. Kwan, Systematic review of the barriers affecting medication adherence in older adults, Geriatr. Gerontol. Int. 16 (10) (2016) 1093-1101, https://doi.org/10.1111/ggi.12616.

[12] J.R. Jardim, O.A. Nascimento, Medical sciences the importance of inhaler adherence to prevent, Med. Sci. 7 (54) (2019) 1-11.

[13] J.L. López-Campos, E.Q. Gallego, L.C. Hernández, Status of and strategies for improving adherence to copd treatment, Int. J. COPD 14 (2019) 1503-1515, https://doi.org/10.2147/COPD.S170848.

[14] S. Amin, M. Soliman, A. McIvor, A. Cave, C. Cabrera, Understanding patient perspectives on medication adherence in asthma: a targeted review of qualitative studies, Patient Prefer. Adherence 14 (2020) 541-551, https://doi.org/10.2147/ PPA.S234651.

[15] A.L. Dima, G. Hernandez, O. Cunillera, M. Ferrer, M. De Bruin, Asthma inhaler adherence determinants in adults: systematic review of observational data, Eur. Respir. J. 45 (4) (2015) 994-1018, https://doi.org/10.1183/09031936.00172114.

[16] R. Normansell, K.M. Kew, E. Stovold, Interventions to improve adherence to inhaled steroids for asthma, Cochrane Database Syst. Rev. 4 (4) (2017), https:// doi.org/10.1002/14651858.CD012226.pub2.

[17] J. Vestbo, et al., Adherence to inhaled therapy, mortality and hospital admission in COPD, Thorax 64 (11) (2009) 939-943, https://doi.org/10.1136/ thx.2009.113662.

[18] J.A.S. Constança Monteiro, Tiago Maricoto, Filipe Prazeres, Pedro Augusto Simões, "PROSPERO protocol_Determining Factors for Inhaled adherence_Final, PROSPERO, Portugal, 2020, p. 6 [Online]. Available: https://www.crd.york.ac.uk /prospero/display_record.php?ID=CRD42020220805\%0A\%0A.

[19] M.J. Page, et al., The PRISMA 2020 statement: an updated guideline for reporting systematic reviews, Syst. Rev. 10 (1) (2021) 1-15, https://doi.org/10.1186/ s13643-021-01626-4.

[20] D.F. Stroup, et al., Meta-analysis of observational studies in epidemiology, JAMA 283 (15) (2000), 2008-12.

[21] D. Moher, et al., Preferred reporting items for systematic review and meta-analysis protocols (PRISMA-P) 2015 statement, Syst. Rev. 4 (1) (2015) 1-9.

[22] R.L. Morgan, et al., A risk of bias instrument for non-randomized studies of exposures: a users' guide to its application in the context of GRADE, Environ. Int. 122 (2019) 168-184, https://doi.org/10.1016/j.envint.2018.11.004. November 2018.

[23] Critical Appraisal Skills Programme, CASP (Checklist Systematic Review), 2018, 1994.

[24] T.J. VanderWeele, P. Ding, Sensitivity analysis in observational research: introducing the E-Value, Ann. Intern. Med. 167 (4) (2017) 268-274, https://doi. org/10.7326/M16-2607.

[25] A. Smith, J.A. Krishnan, A. Bilderback, K.A. Riekert, C.S. Rand, S.J. Bartlett, Depressive symptoms and adherence to asthma therapy after hospital discharge, Chest 130 (4) (2006) 1034-1038, https://doi.org/10.1378/chest.130.4.1034.

[26] J. Voorham, et al., Does co-payment for inhaler devices affect therapy adherence and disease outcomes? A historical, matched cohort study, Pragmatic Obs. Res. 8 (2017) 31-41, https://doi.org/10.2147/por.s132658.

[27] M. Takemura, et al., Relationships between repeated instruction on inhalation therapy, medication adherence, and health status in chronic obstructive pulmonary disease, Int. J. COPD 6 (1) (2011) 97-104, https://doi.org/10.2147/COPD. S16173.

[28] D. Ponieman, J.P. Wisnivesky, H. Leventhal, T.J. Musumeci-Szabó, E.A. Halm, Impact of positive and negative beliefs about inhaled corticosteroids on adherence in inner-city asthmatic patients, Ann. Allergy Asthma Immunol. 103 (1) (2009) 38-42, https://doi.org/10.1016/S1081-1206(10)60141-X.

[29] M.P. Celano, et al., Treatment adherence among low-income, african american children with persistent asthma, J. Asthma 47 (3) (2010) 317-322, https://doi. org/10.3109/02770900903580850.

[30] A.J. Apter, et al., The association of health literacy with adherence and outcomes in moderate-severe asthma, J. Allergy Clin. Immunol. 132 (2) (2013) 321-327, https://doi.org/10.1016/j.jaci.2013.02.014.

[31] I. Sulaiman, et al., Objective assessment of adherence to inhalers by patients with chronic obstructive pulmonary disease, Am. J. Respir. Crit. Care Med. 195 (10) (2017) 1333-1343, https://doi.org/10.1164/rccm.201604-07330C.

[32] A.P. Yu, et al., Therapy persistence and adherence in patients with chronic obstructive pulmonary disease: multiple versus single long-acting maintenance inhalers, J. Med. Econ. 14 (4) (2011) 486-496, https://doi.org/10.3111/ 13696998.2011.594123.

[33] Q.M. van Dellen, K. Stronks, P.J.E. Bindels, F.G. Öry, W.M.C. van Aalderen, Adherence to inhaled corticosteroids in children with asthma and their parents, Respir. Med. 102 (5) (2008) 755-763, https://doi.org/10.1016/j. rmed.2007.12.005.

[34] B.D. De Smet, S.R. Erickson, D.M. Kirking, Self-reported adherence in patients with asthma, Ann. Pharmacother. 40 (3) (2006) 414-420, https://doi.org/10.1345/ aph.1G475.

[35] A.D. Federman, et al., Self-management behaviors in older adults with asthma: associations with health literacy, J. Am. Geriatr. Soc. 62 (5) (2014) 872-879, https://doi.org/10.1111/jgs.12797.

[36] J. Andres, A. Hellmann, T. Hering, R. Hövelmann, M. Weber, Therapieadhärenz bei Asthma bronchiale - versorgung unter Alltagsbedingungen in der pneumologischen Praxis, Pneumologie 68 (5) (2014) 315-321, https://doi.org/10.1055/s-00341365285.
[37] V. Plaza, et al., Impact of patient satisfaction with his or her inhaler on adherence and asthma control, Allergy Asthma Proc. 39 (6) (2018) 437-444, https://doi.org/ 10.2500/aap.2018.39.4183.

[38] D. Ierodiakonou, et al., Adherence to inhalers and comorbidities in COPD patients. A cross-sectional primary care study from Greece, BMC Pulm. Med. 20 (1) (2020) 1-10, https://doi.org/10.1186/s12890-020-01296-3.

[39] M. Olszanecka-Glinianowicz, A. Almgren-Rachtan, The adherence and illness perception of patients diagnosed with asthma or chronic obstructive pulmonary disease treated with polytherapy using new generation Cyclohaler, Postep. Dermatologii i Alergol. 31 (4) (2014) 235-246, https://doi.org/10.5114/ pdia.2014.45070.

[40] B. Sleath, et al., Reported problems and adherence in using asthma medications among adolescents and their caregivers, Ann. Pharmacother. 52 (9) (2018) 855-861, https://doi.org/10.1177/1060028018766603.

[41] S.L. Janson, G. Earnest, K.P. Wong, P.D. Blanc, Predictors of asthma medication nonadherence, Heart \& Lung J. Acute Crit. Care 37 (3) (2008) 211-218, https:// doi.org/10.1016/j.hrtlng.2007.05.014.

[42] O. Turan, P.A. Turan, A. Mirici, Parameters affecting inhalation therapy adherence in elderly patients with chronic obstructive lung disease and asthma, Geriatr. Gerontol. Int. 17 (6) (2017) 999-1005, https://doi.org/10.1111/ggi.12823.

[43] A. Zucchelli, et al., Adherence to COPD free triple inhaled therapy in the real world: a primary care based study, Clin. Res. J. 14 (8) (2020) 732-739, https://doi. org/10.1111/crj.13190.

[44] C.C. Emilio, et al., Is a low level of education a limiting factor for asthma control in a population with access to pulmonologists and to treatment? J. Bras. Pneumol. 45 (1) (2019) 1-5, https://doi.org/10.1590/1806-3713/e20180052.

[45] D. Price, D.L. Keininger, B. Viswanad, M. Gasser, S. Walda, F.S. Gutzwiller, Factors associated with appropriate inhaler use in patients with COPD - lessons from the REAL survey, Int. J. COPD 13 (2018) 695-702, https://doi.org/10.2147/COPD. S149404.

[46] J. George, D.C.M. Kong, R. Thoman, K. Stewart, Factors associated with medication nonadherence in patients with COPD, Chest 128 (5) (2005) 3198-3204, https:// doi.org/10.1378/chest.128.5.3198.

[47] K.C. Chiu, et al., Patients' beliefs and behaviors related to treatment adherence in patients with asthma requiring maintenance treatment in Asia, J. Asthma 51 (6) (2014) 652-659, https://doi.org/10.3109/02770903.2014.898772.

[48] M. Takemura, et al., Optimal cut-offvalue and clinical usefulness of the Adherence Starts with Knowledge-12 in patients with asthma taking inhaled corticosteroids, J. Thorac. Dis. 9 (8) (2017) 2350-2359, https://doi.org/10.21037/ jtd.2017.06.115.

[49] E. Barja-Martínez, S. Casas-González, A.F. Simón-López, C. Mancheño-Ovejero, M. L.G. Padial-de la Cruz, Adherence to inhaled therapy in the outpatient setting, Enfermería Clínica (English Ed. 29 (1) (2019) 34-38, https://doi.org/10.1016/j. enfcle.2018.09.002.

[50] T. Erdogan, Evaluating nonadherence to preventer inhaler therapy in severe asthmatic patients receiving omalizumab, Clin. Res. J. (2020) 1-6, https://doi.org/ 10.1111/crj.13252. April 2019.

[51] J. Darbà, G. Ramírez, A. Sicras, P. Francoli, S. Torvinen, R. Sánchez-De La Rosa The importance of inhaler devices: the choice of inhaler device may lead to suboptimal adherence in COPD patients, Int. J. COPD 10 (1) (2015) 2335-2345, https://doi.org/10.2147/COPD.S90155.

[52] J.C. Huetsch, J.E. Uman, E.M. Udris, D.H. Au, Predictors of adherence to inhaled medications among veterans with COPD, J. Gen. Intern. Med. 27 (11) (2012) 1506-1512, https://doi.org/10.1007/s11606-012-2130-5.

[53] R. O'Conor, et al., Effects of health literacy and cognitive abilities on COPD selfmanagement behaviors: a prospective cohort study, Respir. Med. 160 (2019) 1-7, https://doi.org/10.1016/j.rmed.2019.02.006. November 2018.

[54] M. Takemura, et al., Repeated instruction on inhalation technique improves adherence to the therapeutic regimen in asthma, J. Asthma 47 (2) (2010) 202-208, https://doi.org/10.3109/02770900903581692.

[55] K. Wells, et al., Race-ethnic differences in factors associated with inhaled steroid adherence among adults with asthma, Am. J. Respir. Crit. Care Med. 178 (12) (2008) 1194-1201, https://doi.org/10.1164/rccm.200808-12330C.

[56] V. Plaza, et al., Assessing adherence by combining the test of adherence to inhalers with pharmacy refill records, J Investig. Allergol. Clin. Immunol. 31 (1) (2019) 1-23, https://doi.org/10.18176/jiaci.0461.

[57] G. Kardas, M. Panek, P. Kuna, P. Kardas, Primary non-Adherence to inhaled medications measured with e-prescription data from Poland, Clin. Transl. Allergy 10 (1) (2020) 1-8, https://doi.org/10.1186/s13601-020-00346-7.

[58] C.V. Chambers, L. Markson, J.J. Diamond, L. Lasch, M. Berger, Health beliefs and compliance with inhaled corticosteroids by asthmatic patients in primary care practices, Respir. Med. 93 (2) (1999) 88-94, https://doi.org/10.1016/S0954-6111 (99)90296-2.

[59] Y. Lacasse, H. Archibald, P. Ernst, L.P. Boulet, Patterns and determinants of compliance with inhaled steroids in adults with asthma, Cancer Res. J. 12 (4) (2005) 211-217, https://doi.org/10.1155/2005/375454.

[60] M. Humenberger, et al., Adherence to inhaled therapy and its impact on chronic obstructive pulmonary disease (COPD), BMC Pulm. Med. 18 (1) (2018) 1-6, https://doi.org/10.1186/s12890-018-0724-3.

[61] R. O'Conor, et al., Health literacy, cognitive function, proper use, and adherence to inhaled asthma controller medications among older adults with asthma, Chest 147 (5) (2015) 1307-1315, https://doi.org/10.1378/chest.14-0914.

[62] V. Plaza, et al., Differences in adherence and non-adherence behaviour patterns to inhaler devices between COPD and asthma patients, COPD J. Chronic Obstr. Pulm. Dis. 13 (5) (2016) 547-554, https://doi.org/10.3109/15412555.2015.1118449. 
[63] A.J. Apter, S.T. Reisine, G. Affleck, E. Barrows, R.L. ZuWallack, Adherence with twice-daily dosing of inhaled steroids: socioeconomic and health-belief differences, Am. J. Respir. Crit. Care Med. 157 (6) (1998) 1810-1817, https://doi.org/ 10.1164/ajrccm.157.6.9712007. PART I.

[64] B. Bender, H. Milgrom, C. Rand, L. Ackerson, Psychological factors associated with medication nonadherence in asthmatic children, J. Asthma 35 (4) (1998) 347-353, https://doi.org/10.3109/02770909809075667.

[65] D. Aggarwal, M. Bhardwaj, B. Singh, A.K. Janmeja, Factors determining adherence to inhaled corticosteroids in bronchial asthma patients at a tertiary care hospital in India, J. Clin. Diagn. Res. 11 (12) (2017) OC14-OC18, https://doi.org/10.7860/ JCDR/2017/32568.10988.

[66] S. Tavasoli, H. Heidarnazhad, A. Kazemnejad, Factors affecting patients' compliance to metered-dose inhaler drugs in two asthma clinics in Tehran, Iran, Iran. J. Allergy, Asthma Immunol. 5 (4) (2006) 187-193, 05.04/ijaai.187193.

[67] J.M. Foster, et al., Identifying patient-specific beliefs and behaviours for conversations about adherence in asthma, Intern. Med. J. 42 (6) (2012), https:// doi.org/10.1111/j.1445-5994.2011.02541.x.

[68] L.K. Williams, et al., Race-ethnicity, crime, and other factors associated with adherence to inhaled corticosteroids, J. Allergy Clin. Immunol. 119 (1) (2007) 168-175, https://doi.org/10.1016/j.jaci.2006.09.029.

[69] E. Vasbinder, et al., The association of ethnicity with electronically measured adherence to inhaled corticosteroids in children, Eur. J. Clin. Pharmacol. 69 (3) (2013) 683-690, https://doi.org/10.1007/s00228-012-1380-9.

[70] K.B. Sriram, M. Percival, Suboptimal inhaler medication adherence and incorrect technique are common among chronic obstructive pulmonary disease patients, Chron. Respir. Dis. 13 (1) (2016) 13-22, https://doi.org/10.1177/ 1479972315606313.

[71] B. Bhattarai, R. Walpola, A. Mey, S. Anoopkumar-Dukie, S. Khan, Barriers and strategies for improving medication adherence among people living with copd: a systematic review, Respir. Care 65 (11) (2020) 1738-1750, https://doi.org/ 10.4187/respcare.07355.

[72] A. Torres-Robles, E. Wiecek, F.S. Tonin, S.I. Benrimoj, F. Fernandez-Llimos, V. Garcia-Cardenas, Comparison of interventions to improve long-term medication adherence across different clinical conditions: a systematic review with network meta-analysis, Front. Pharmacol. 9 (2018), https://doi.org/10.3389/ fphar.2018.01454. December.

[73] P. Kardas, P. Lewek, M. Matyjaszczyk, Determinants of patient adherence: a review of systematic reviews, Front. Pharmacol. 4 (July) (2013) 1-16, https://doi.org/ 10.3389/fphar.2013.00091. JUL.
[74] M.H.H. Cheen, Y.Z. Tan, L.F. Oh, H.L. Wee, J. Thumboo, Prevalence of and factors associated with primary medication non-adherence in chronic disease: a systematic review and meta-analysis, Int. J. Clin. Pract. 73 (6) (2019) 1-18, https://doi.org/ 10.1111/ijcp.13350.

[75] M.S. Charles, C.M. Blanchette, H. Silver, D. Lavallee, A.A. Dalal, D. Mapel, Adherence to controller therapy for chronic obstructive pulmonary disease: a review, Curr. Med. Res. Opin. 26 (10) (2010) 2421-2429, https://doi.org/ 10.1185/03007995.2010.516284.

[76] C.B. Bårnes, C.S. Ulrik, Asthma and adherence to inhaled corticosteroids: current status and future perspectives, Respir. Care 60 (3) (2015) 455-468, https://doi. org/10.4187/respcare.03200.

[77] M. Engelkes, H. Janssens, J. de Jongste, M. Sturkenboom, K. Verhamme, Medication adherence and the risk of severe asthma exacerbations: a systematic review, Eur. Respir. J. 45 (2) (2015) 396-407, https://doi.org/10.1183/ 09031936.00075614.

[78] M. Hassan, S.E. Davies, S.P. Trethewey, A.H. Mansur, Prevalence and predictors of adherence to controller therapy in adult patients with severe/difficult-to-treat asthma: a systematic review and meta-analysis, J. Asthma 57 (12) (2020) 1379-1388, https://doi.org/10.1080/02770903.2019.1645169.

[79] M. McGuire, Iuga, Adherence and health care costs, Risk Manag. Healthc. Pol. (Feb. 2014) 35, https://doi.org/10.2147/RMHP.S19801.

[80] H. Esan, J. Shuter, A.H. Weinberger, The relationship of anxiety and smoking behaviors to medication adherence among cigarette smokers living with HIV, Addict. Behav. 90 (Mar. 2019) 301-305, https://doi.org/10.1016/j. addbeh.2018.10.051.

[81] J. Shuter, S. Bernstein, Cigarette smoking is an independent predictor of nonadherence in HIV-infected individuals receiving highly active antiretroviral therapy, Nicotine Tob. Res. 10 (4) (Apr. 2008) 731-736, https://doi.org/10.1080/ 14622200801908190.

[82] L.J. Anderson, et al., A systematic overview of systematic reviews evaluating medication adherence interventions, Am. J. Heal. Pharm. 77 (2) (2020) 138-147, https://doi.org/10.1093/ajhp/zxz284.

[83] W. Shahin, G.A. Kennedy, I. Stupans, The impact of personal and cultural beliefs on medication adherence of patients with chronic illnesses: a systematic review, Patient Prefer. Adherence 13 (2019) 1019-1035, https://doi.org/10.2147/PPA. S212046. 